\title{
The role of hemodynamic parameters in chronic heart failure diagnosis in children
}

\author{
Victoria GROSU, doctor habilitat in biology sciences, associate professor \\ Department of Pediatrics, "Nicolae Testemitanu" State University of Medicine and Pharmacy \\ Chisinau, the Republic of Moldova
}

\begin{abstract}
Despite the achievements in this field in recent years, concerning the chronic myocardial dysfunctions, pathogenesis, diagnosis and treatment management, the chronic heart failure continues to hold preeminence in the structure of cardiovascular complications and remains now one of the leading causes of invalidity of young contingent and infant mortality, presenting one of the most pressing public health problems. This requires looking for new methods of noninvasive early diagnosis with application of modern laboratory and investigational tests facilitating the identification in preclinical stages of chronic myocardial dysfunctions $(C M D)$ by assessment of new biomarkers of myocardial injury.

Objectives of the study. Hemodynamic parameters changes estimation in the evolution of chronic heart failure in children.

Material and methods. The study included 233 patients with chronic heart failure, of which 78 were children and adolescents aged 10-17 years with CMD secondary to arterial hypertension. The diagnosis of arterial hypertension was established according to the ESA (European Society of Cardiology, 2009) recommendations, but the assessment of clinical manifestations of chronic heart failure was evaluated according to NYHA (New York Heart Association classification). The estimation of hemodynamic parameters was performed using echocardiography method using "Ultramark-8" (US device A) under Mand Doppler.

Obtained results. In patients with arterial hypertension the types of concentric hypertrophy of left ventricular myocardial index mass (IMMVS) $-59.2 \pm 14.2$ and relative $L V$ wall thickness (GRPVS) $-0.46 \pm 0.22$ $\mathrm{cm}$ were assessed. It is important to note that the study assessed the type of eccentric hypertrophy with IMMVS 66.4 \pm 7.6 and $0.44 \pm 0.25 \mathrm{~cm}$ GRPVS. Concentric geometric remodeling variant noted IMMVS average value of $48.4 \pm 4.2$ and GRPVS- $0.44 \pm 0.21 \mathrm{~cm}$. Identification of LV hypertrophy, increased GRPVS, IMMVS increase, the MMV based on echocardiographic examination of the heart reveals the geometric performance called "pathological remodeling" which actually confirms the occurrence of chronic myocardial dysfunction in patients with CHF secondary hypertension.

Conclusions. Early identification of changes of cardiac performance parameters (MMV, IMMVS, GRPVS, HVS) under the conditions of the persistent tensional over estimation, reveals the early development of types of early myocardial pathological left ventricular remodeling and facilitates the options and diagnostic criteria of the heart remodeling in children with CMD secondary arterial hypertension in order to choose a selected pathogenetic treatment.
\end{abstract}

Keywords: chronic heart failure, arterial hypertension, cardiac remodeling 


\section{INTRODUCTION}

Currently, chronic heart failure (CHF), polyetiologic syndrome is defined as a dynamic process where neuroendocrine changes, hemodynamic factors, causing myocardial remodeling and functionality alteration, changes in metabolism and other disorders.

Prevalence of chronic heart failure is increasing worldwide, having a poor prognosis and reducing survival perspective, altering the quality of life, causing disability and being associated with increased morbidity.

Chronic dysfunctions of the myocardium (CDM) is the final event of the development of any cardiovascular diseases and maintain constantly at a high incidence morbidity due to cardiovascular diseases. Obviously, progressive incidence, complexity of the clinical picture of the syndrome of heart failure study places it on top of contemporary medicine $(1,2)$. Arterial hypertension (AHT) is currently considered one of the most common pathologies of the cardiovascular system and one of the risk factors of atherosclerosis and chronic myocardial dysfunctions $(3,4)$. Essential hypertension is accompanied by metabolic disorders, hypertriglyceridemia, hyperinsulinemia, excessive fat storage in adipocytes in obese patients as well as other factors.

To assess the degree and prognosis of arterial hypertension, it is important to use early diagnosis, first of all the signs of heart remodeling and changes in the arterial wall. Management of cardiovascular diseases diagnosis usually includes two directions - invasive and non-invasive diagnosis, the last being meant to strengthen clinical and therapeutic possibilities.

The AHT under the action of various pathogenic links disorders of various organs' functions - of heart, vessels, kidneys, brain, eyes occur. Thus, it was shown that, the most common sign of target organ damage of arterial hypertension is left ventricular myocardial hypertrophy with its geometric remodeling, where blood pressure expression and prolonged overvalues duration have a major role in the development $(5,6)$. Currently the development of left ventricle myocardium hypertrophy in AHT is appreciated due to structural adaptation of the myocardium as a response to increased physiological function, but during blood pressure overvalues prolongation, adaptation changes turn in to non adaptation ones.

\section{OBJECTIVES}

Estimating changes in hemodynamic parameters in the evolution of chronic heart failure in children.

\section{MATERIALS AND METODS}

The study included 233 patients with chronic heart failure (CHF) who were selected from 78 patients with chronic myocardial dysfunctions secondary to arterial hypertension (AHT) aged 10-17 years. The diagnosis of AHT was established according to the recommendations of SEC (2009) and the clinical manifestations estimation of CHF was assessed according to NYHA (New York Heart Association) classification. Determination of hemodynamic parameters was performed using echocardiography method using "Ultramark-8" (USA) device with $\mathrm{M}$ and Doppler functions.

From the general study group 78 patients with DCM secondary to arterial hypertension 38 boys $(48.7 \%)$ and 40 girls $(51.3 \%)$ were selected who constituted two research groups. The study group I was formed from 41 children with a mean age of $15.1 \pm 0.24$ years, who received treatment with converting enzyme inhibitor of angiotensin Captopril the dosage being calculated according to the body weight, $1 \mathrm{mg} / \mathrm{kg} / 24$ hours 3 times. During the clinical trial was used a combined therapy with antagonist receptors of aldosterone Spironolactone in doses according to the body weight $-1 \mathrm{mg} /$ $\mathrm{kg}$ in 1-2 doses/24 hours. Lot II study included 37 children, mean age $13.4 \pm 0.26$ years, with CHF secondary to arterial hypertension who received treatment with inhibitor converting enzyme of angiotensin Enalapril in the dosage of $0.06 \mathrm{mg} / \mathrm{kg} /$ day every 24 hours. The control group consisted of 85 healthy children without signs of CHF the echocardiographic parameters were estimated and referred to laboratory indices. The treatment of patients included in the study was initiated only in hospital conditions with prompt monitoring clinical, hemodynamic indicators, including FCC values, pulse, TA, diuresis. Duration of treatment in these patients is individually programmed as tolerated preparation, improving clinical indicators, hemodynamic, regression of clinical signs of $\mathrm{CHF}$, improving the exercise tolerance, and biochemical indices and hormonal normalization. Clinical data were objectified to support laboratory investigations: ECG, echocardiography and ambulatory 24-hours automatic monitoring of TA. In the 
general examination of patients following the investigations additional tools was carried out: ultrasound of internal organs and the urinary system organs, urography and/vein as clinically indicated, retinoscope. The diagnosis of arterial hypertension was confirmed by the following methods:

1) on the basis of clinical data - AHT assessing determining repeated dynamic tensiometry of upper limbs and lower limbs and AHT level check to exclude coarctation of the aorta; general clinical symptoms nausea, headache, visual changes, cardiac algias, sensations of choking and retrosternal pain and dyspnea-like symptoms, heart palpitations, CHF characteristic fatigue classified as NYHA. Level arterial hypertension was appreciated by percentile method ( $T A \geq 97.5$ percentile) using percentile tables developed according to age, sex, height and weight of the patient based on the recommendations set out in the guidelines adopted by the European Society of Cardiology in 2009 (3);

2) based on instrumental examinations: a) ECG standard - ECG abnormalities identification, particularly of ventricular myocardial hypertrophy signs, abnormal ventricular electrical systole QT (ms) prolongation, heart rhythm disturbances, changes in the remodeling process of left ventricle myocardium, b) radiographs of the chest organs - constituting radiological changes of the heart and great vessels shadow with ICT increase; c) echocardiography - with the appreciation of all hemodynamic parameters including heart chamber diameters, intracavitary volumes (VTDVS, VTSVS) signs of myocardial hypertrophy, LV, LV wall thickness. Ejection fraction (EF) determined during EcoCG notes the systolic function of the heart. EF index $<25 \%$ indicates a severely impaired heart function which corresponds to a serious condition of the patient with signs of CHF functional class III-IV NYHA. The echocardiography examination is important to assess the presence and severity of heart failure, it allows to distinguish between systolic and diastolic forms of chronic heart failure. It should be noted that in the systolic dysfunction ejection fraction (EF) $<45 \%$, the diastolic dysfunction ejection fraction may be - EF> 50\% (5). The severity of chronic heart failure in the clinical examination is difficult to assess objectively, and this examinations - the key is EcoCG; d) automatic ambulatory monitoring $\mathrm{AH} / 24$ hours $\mathrm{AH}$ diurnal and nocturnal appreciation profile.

Exclusion criteria from the study in the general group of patients with arterial hypertension were the following diseases: coarctation of the aorta, pheochromocytoma, adrenal pathology (Cushing's, Conn, Liddle syndrome), hyperthyroidism, intracranial hypertension, hypercalcemia, reactive mental states, glomerulonephritis, Fanconi hypertension, renal polichistosis, hydronephrosis, renal hypoplasia, steroids medication, smoking, drugs, alcohol, intoxication with drugs.

The study's raw materials were processed using the computer program "Statistical Package for the Social Science" by means of variational descriptive analysis. Statistical dependence between qualitative parameters was presented in contingency tables and to verify the hypothesis of independence of rows and columns "THI" $\left(X^{2}\right)$ criterion was used.

To estimate the significant differences in the averages of two groups we used Student criterion. Testing group dynamics parameters was performed by T-test coherent selection criteria. To estimate the significant differences in both groups positive samples values were used U-Fischer criterion.

\section{RESULTS}

The estimation of general clinical status was based on functional class of CHF. Distribution of $\mathrm{CHF}$ functional class in patients with arterial hypertension was the following: in study group I in patients with arterial hypertension in $11.5 \%$ of cases was assessed NYHA functional class I and in $54.6 \%$ of patients in NYHA functional class II; functional class NYHA III - in $33.9 \%$ of cases; in study group II in patients with arterial hypertension - in $13.2 \%$ of cases NYHA functional class I was appreciated, $62.8 \%$ cases - NYHA functional class II, and $24 \%$ clinical cases of severe heart failure - NYHA functional class III.

As clinical symptoms in patients with arterial hypertension prevailed the following signs: tachycardia (heart palpitations) - in 66 patients $(84.6 \%)$ cases ( $p<0.01)$, headache - in 39 patients $(50 \%)$ cases $(p<0.001)$, fatigue - from 71 patients $(91 \%)$. The dynamics of functional values of hemodynamic parameters in 6 months following treatment of the estimated results shown in Table 1.

As it can be seen in Table 1 the values of the hemodynamic parameters of systemic systolic blood pressure, sAHT, dAHT noted significant differences compared to the control group initially at the admission in the study in both investigated groups $(p<0.001)$ with a 
significant reduction in blood pressure in $d y-$ namics treatment over 6 months of observation without differences between them, but it was recorded in relation with control group variation and in relation to the initial indicators ( $p$ $<0.001$ ). Average blood pressure values in both groups identically increased so that systolic AHT increased initially in group I by $53 \%$, and dAHT - with $77 \%$ respectively, in group II SAHT increased by $53 \%$, and $\mathrm{dAHT}$ by $79 \%$ compared with the control group. Average frequency of cardiac rate (FCR) increased initially in group I by $23 \%$, while in group $11-47 \%$ compared to the control group with subsequent normalization in the observational period, presenting authentic dynamic group differences $(p<0.001)$. FCR indices in both groups initially estimated values significantly different from the control group ( $p$ $<0.001)$. Cardiac performance parameter values (LVMM, LVMMI) showed significant increases in both groups compared with control group both at the stage of inclusion in the study, and at 6 months of observation $(p<0.001)$. Thus, LVMM initially increased by $103 \%$ in group I and in group II with $128 \%$ after 6 months remained increased by $91 \%$ in group I and in group - II - 108\% respectively. LV myocardial mass index changes in both groups were distributed so that LVMMI initially in both groups increased by $78 \%$ compared with control group, and in 6 months the values had cast changes in group I by $86 \%$ and group $I I-91 \%$ respectively.

Comparative analysis of electrocardiographic abnormalities at an early stage in patients with arterial hypertension showed conclusive true differences by the presence of electrocardiographic signs typical of chronic myocardial dysfunctions, such as changes in the QRS pattern and $T$ wave, including suggestive signs of remodeling processes disturbances - various changes of ST-T and QT prolongation up to 40"42". Electrocardiographic signs of LV hypertrophy of the myocardium are characteristic for patients with arterial hypertension, significantly presented between groups $(p<0.01)$ and confirms the onset of chronic myocardial dysfunction in the study group.

Electrophysiological peculiarities documented by ECG examination in observational dynamics of the studied groups patients are shown in Table 2. Once the data presented in this table at six months period of clinical observation, improvements in heart rate are noticed returning to sinus rhythm in $79.4 \%$ cases, sinus tachycardia reduction in $55.1 \%$ cases of QT interval duration $27.7 \mathrm{~ms}$, improving the QRST complex morphology in $71.7 \%$ of cases, ventricular extrasystole disappearance, reducing the frequency of supraventricular extrasystole in $15.3 \%$ of cases, improvement in LV myocardium remodeling processes in $98.7 \%$ of cases and reduction of myocardial remodeling of LV hypertrophy in $71.7 \%$ of cases.

It is proven that changing the geometry of the LV myocardium anticipates the development of myocardial hypertrophy. LV geometric model is estimated on the basis of left ventricular myocardial mass index and relative wall thickness index ventricle. LV relative wall thickness was calculated as the ratio between the thickness of the volume of the left ventricle myocardium. The criterion of relative increase in LV wall thickness, which identifies left ventricular remodeling in adult patients ranges from 0.40 to 0.44 (5).

Theme an value of left ventricle wall relative thickness, in the current study, is higher in adolescents with stable arterial hypertension $(0.41$ \pm 0.25 ), whereas in patients with labile AHT is $0.39 \pm 0.12$, which actually was not different from the control group $(0.37 \pm 0.02)$.

Thus, the index of LV wall relative thickness with the value which exceeded more than 0.44 was considered in $28.5 \%$ of cases (8 patients) of the total $(n=28)$ of patients with stable arterial hypertension with diastolic dysfunction documented by echocardiography, rarely in patients with labile AHT in $14.2 \%$ of cases (4 patients) and was not determined in adolescents with normal arterial pressure from the control group.

Heart remodeling variants and diagnostic criteria in patients with CHF secondary to arterial hypertension are presented in Table 3.

\section{DISCUSSION}

Clinical paraclinical events evolution in the present study was the following: patients who had received combination therapy (Captopril and Spironolactone) showed a significant improvement both clinically reducing clinical functional signs of heart insufficiency and a significant reduction of blood pressure resulting in a genuine difference between groups. At 6 months period of clinical observation patients receiving Enalapril (group II of study) have improved their performance, but obviously have yielded to the I group with a significant difference according to the performance indexes of heart as the LV myocardial mass ( $\mathrm{g}$ ) value and body mass index of the LV myocardium (LVMMI). 
TABLE 1. Evolution of hemodynamic parameters in children with arterial hypertension

\begin{tabular}{|c|c|c|c|c|c|}
\hline Index & Group & Initially & 6 months & p & Control group \\
\hline \multirow{3}{*}{$\mathrm{sAHT}, \mathrm{mmHg}$} & 1 & $\begin{array}{c}158,0 \pm 3,2 * * * \\
(153 \%)\end{array}$ & $\begin{array}{c}122,3 \pm 2,0 * * * \\
(118 \%)\end{array}$ & $<0,001$ & \multirow{3}{*}{$103,6 \pm 0,3$} \\
\hline & II & $\begin{array}{c}158,6 \pm 3,4^{* * *} \\
(153 \%)\end{array}$ & $\begin{array}{c}124,0 \pm 2,1 * * * \\
(120 \%)\end{array}$ & \multirow[t]{2}{*}{$<0,001$} & \\
\hline & $\mathrm{P}$ & $>0,05$ & $>0,05$ & & \\
\hline \multirow{3}{*}{$\begin{array}{l}\mathrm{dAHT}, \\
\mathrm{mm} \mathrm{Hg}\end{array}$} & 1 & $\begin{array}{c}96,3 \pm 1,35 * * * \\
(177 \%) \\
\end{array}$ & $\begin{array}{c}80,9 \pm 1,3 * * * \\
(148 \%)\end{array}$ & $<0,001$ & \multirow{3}{*}{$54,6 \pm 2,4$} \\
\hline & II & $\begin{array}{c}97,6 \pm 1,5 * * * \\
(179 \%) \\
\end{array}$ & $\begin{array}{c}82,6 \pm 1,2 * * * \\
(151 \%)\end{array}$ & \multirow[t]{2}{*}{$<0,001$} & \\
\hline & $\mathrm{P}$ & $>0,05$ & $>0,05$ & & \\
\hline \multirow{3}{*}{$\mathrm{FCR}, \mathrm{b} / \mathrm{min}$} & I & $\begin{array}{c}104,0 \pm 4,1^{* * *} \\
(123 \%) \\
\end{array}$ & $\begin{array}{c}77,0 \pm 1,4^{*} \\
(91 \%) \\
\end{array}$ & $<0,001$ & \multirow{3}{*}{$84,5 \pm 3,4$} \\
\hline & II & $\begin{array}{c}124,2 \pm 4,1^{* * *} \\
(147 \%)\end{array}$ & $\begin{array}{c}79,6 \pm 1,6 \\
(94 \%)\end{array}$ & \multirow[t]{2}{*}{$<0,001$} & \\
\hline & $p$ & $<0,01$ & $>0,05$ & & \\
\hline \multirow{3}{*}{ LVMMI, g/m² } & I & $\begin{array}{c}82,3 \pm 2,2 * * * \\
(178 \%)\end{array}$ & $\begin{array}{c}86,9 \pm 3,8 * * * \\
(186 \%)\end{array}$ & $>0,05$ & \multirow{3}{*}{$46,5 \pm 1,3$} \\
\hline & ॥ & $\begin{array}{c}82,4 \pm 1,4 * * * \\
(178 \%)\end{array}$ & $\begin{array}{c}88,9 \pm 4,0 * * * \\
(191 \%)\end{array}$ & \multirow[t]{2}{*}{$>0,05$} & \\
\hline & $p$ & $>0,05$ & $>0,05$ & & \\
\hline \multirow{3}{*}{ LVMM, g } & I & $\begin{array}{c}162,7 \pm 7,1^{* * *} \\
(203 \%) \\
\end{array}$ & $\begin{array}{c}157,6 \pm 7,0 * * * \\
(191 \%)\end{array}$ & $>0,05$ & \multirow{3}{*}{$82,6 \pm 4,2$} \\
\hline & II & $\begin{array}{c}187,0 \pm 12,8 * * * \\
(228 \%)\end{array}$ & $\begin{array}{c}171,3 \pm 10,7^{* * *} \\
(208 \%)\end{array}$ & \multirow[t]{2}{*}{$>0,05$} & \\
\hline & $p$ & $>0,05$ & $>0,05$ & & \\
\hline
\end{tabular}

Note: Statistically significant differences compared with the indicators of control group: $* p<0.05 ; * * * p<0.01 ; * * * p<0.001$.

TABLE 2. Electrocardiographic changes in patients with chronic myocardial dysfunctions secondary to arterial hypertension

\begin{tabular}{|c|c|c|c|c|c|}
\hline \multirow[t]{2}{*}{ ECG signs } & \multicolumn{2}{|c|}{$\begin{array}{l}\text { Patients with AHT } \\
\text { ( } n=78) \text { initially }\end{array}$} & \multicolumn{2}{|c|}{$\begin{array}{l}\text { Patients with AHT } \\
(n=78) \text { in } 6 \text { months }\end{array}$} & \multirow[t]{2}{*}{ p } \\
\hline & $\mathbf{N}$ & $\%$ & $\mathbf{N}$ & $\%$ & \\
\hline ECG signs - sinus rhythm & 62 & 79,4 & 78 & 100 & $>0,05$ \\
\hline Sinus tachycardia & 43 & 55,1 & 2 & 2,5 & $<0,001$ \\
\hline Sinus bradycardia & 4 & 5,1 & 1 & 1,2 & $<0,05$ \\
\hline Q-T interval duration, $\mathrm{ms}$ & \multicolumn{2}{|c|}{$396,3 \pm 11,2$} & \multicolumn{2}{|c|}{$363,6 \pm 6,4$} & $>0,05$ \\
\hline ST segm. depression, $\mathrm{mm}$ & \multicolumn{2}{|c|}{$1,80 \pm 0,06$} & \multicolumn{2}{|c|}{$0,8 \pm 0,02$} & $<0,001$ \\
\hline $\begin{array}{l}\text { QRST compl. morphology } \\
\text { modification signs }\end{array}$ & 56 & 71,7 & 8 & 10,2 & $<0,001$ \\
\hline Extrasystole, s/ventricular & 12 & 15,3 & 3 & 3,8 & $<0,05$ \\
\hline Ventricular extrasystole & 8 & 10,2 & 1 & 1,2 & $<0,001$ \\
\hline $\begin{array}{l}\text { Repolarization disorders in LV } \\
\text { myocardium }\end{array}$ & 77 & 98,7 & 4 & 5,1 & $<0,001$ \\
\hline LV myocardium hypertrophy & 56 & 71,7 & 24 & 30,7 & $<0,001$ \\
\hline
\end{tabular}

Note: Statistically significant differences between groups - $* p<0.05, * * p<0.01, * * * p<0.001$.

TABLE 3. Types of myocardial left ventricle remodeling in patients with chronic myocardial dysfunctions secondary to arterial hypertension

\begin{tabular}{|l|c|c|}
\hline Geometry of the left ventricle & $\begin{array}{c}\text { Left ventricle's myocardium } \\
\text { index mass (LVMMI, } \mathbf{g} / \mathbf{m}^{\mathbf{2}} \text { ) }\end{array}$ & $\begin{array}{c}\text { Relative thickness of the wall of } \\
\text { the left ventricle (RTWLV, } \mathbf{~ c m})\end{array}$ \\
\hline Normal (control) & $39,5 \pm 1,3$ & $0,37 \pm 0,02$ \\
\hline Concentric hypertrophy & $59,2 \pm 14,2$ & $0,46 \pm 0,22$ \\
\hline Eccentric hypertrophy & $66,4 \pm 7,6$ & $0,44 \pm 0,25$ \\
\hline Concentric remodeling & $48,4 \pm 4,2$ & $0,44 \pm 0,21$ \\
\hline
\end{tabular}


During the research LV myocardium mass index control was calculated in the control group having the value of $39.5 \pm 1.3$, and the relative thickness of the wall of the left ventricle was noted in the amount of $0.37 \pm 0.02$, which corresponds to the data literature (7). In patients with arterial hypertension were assessed the concentric hypertrophy types IMMVS - 59.2 \pm 14.2 and GRPVS $-0.46 \pm 0.22$. It is important to note that during the study the type of eccentric hypertrophy with IMMVS have been assessed 66.4 \pm 7.6 and $0.44 \pm 0.25$ GRPVS. Concentric remodeling geometric variant noted IMMVS average value of $48.4 \pm 4.2$ and GRPVS $-0.44 \pm 0.21$.

On this basis several types of cardiac remodeling were determined: concentric hypertrophy of the LV myocardium, myocardial eccentric hypertrophy and concentric remodeling of the LV myocardium. The type of cardiac remodeling of the left ventricle is currently considered as a major criterion not only for the severity of the disease, but also for prognosis of arterial hypertension. Concentric hypertrophy of the myocardium is considered a predictor of high risk of developing cardiovascular complications. Eccentric hypertrophy is accompanied by an average cardiovascular risk of complications during the adolescent and young adult, and subsequent periods of age $(7,8)$. It is important to note that the occurrence of myocardial remodeling depends on the version arterial hypertension.

The echocardiographic parameters variables evaluation of the obtained LV myocardium indicate geometric performance of the heart called "pathological remodeling" which actually confirms the occurrence of chronic myocardial dysfunctions in patients with CHF secondary to arterial hypertension. Assessing the things previously reported we found out that the ICC secondary to AHT significant changes of electrophysiological parameters of cardiac activity occur, which suggests a disorder of regulatory systems in cardiac electrophysiology and significantly influences the clinical status of patients and the development of chronic myocardial dysfunctions. The changes specificity in ECG parameters in chronic heart failure secondary to arterial hypertension influences the prognosis of patients with an increased risk of sudden death. Therefore it is important to detect the early signs of heart failure in this group of patients and initiate pathogenetic treatment to support the heart'spump function without increasing myocardial oxygen demand. For this it was opportune the choice of preparing group especially of IECA inhibitors either alone or in combination therapy (ACEI + aldosterone receptor antagonist) individually selected due to the patient's clinical indications.

The treatment of chronic myocardial dysfunctions experienced remarkable progress in the last two decades. The hyperactivity modulation of the renin-angiotensin-aldosterone system (by converting enzyme inhibitors and/ or angiotensin receptor blockers, aldosterone receptors antagonists through spironolactone and eplerenone) and the sympatho-adrenergic $\beta$-adrenoblocker with had a strong impact on mortality and morbidity by ICC, AHT in adults (9). Currently, the ICC therapeutic approach in children enrolles strategies defined with certainty from a large number of randomized placebo-controlled multicenter studies aimed at the influence of different remedies on indices of morbidity, mortality and improvement of life quality and prognosis of patients' lives (10-12). Due to the difficulties of specifying diagnostic chronic myocardial dysfunctions in children currently there is no unanimity in the development of treatment options for this condition. Therapeutic options used in the present study carries a high distinction depending on the pathogenetic modules of chronic heart failure syndrome development and confirmed an improvement in clinical and paraclinical long lasting events, influiencing progression of chronic myocardial dysfunctions in children.

\section{CONCLUSIONS}

1. Early identification of myocardium performance parameter schanges (LVMM, LVMMI, RTWLV, LVH), under the conditions of persistent blood pressure over values of the current study, indicate early appearance of types of pathological remodeling of the left ventricle myocardium, and facilitates remodeling options and diagnostic criteria of heart in children with chronic myocardial dysfunctions, secondary to arterial hypertension in order to choose a selected pathogenetic treatment.

2. The results of the study show that patients with arterial hypertension, who received combination therapy with angiotensin converting enzyme inhibitor (Captopril) and aldosterone receptors antagonist (Spironolactone) showed a significant improvement clinically reducing the functional symptoms of heart failure, as well as a significant decrease of blood pressure resulting in a genuine difference between the groups. 
3. In 6 months period, after the observational period in patients treated with angiotensin converting enzyme inhibitor (Enalapril) from the II group study, have clearly improved their performance, but yielded significantly to those in group I of study, who had received a combination therapy with the above mentioned remedies with a true difference according to performance indexes of heart as the LV myocardial mass (g) and LV myocardial mass index.

\section{REFERENCES}

1. Thakur V. et al. Diagnosis and management of fetal heart failure. Can. J. Cardiol. 2013; 29:759-767.

2. Paul F. Kantor et al. Presentation, Diagnosis, and Medical Management of Heart Failure in Children: Canadian Cardiovascular Society Guidelines. Canadian Journal of Cardiology. 2013; 29:1535-52.

3. Empar Lurbe, Cifkova R., Kennedy J. Management of high blood pressure in children and adolescents: recommentations of the European Society of Hypertension. Journal of Hypertension. 2009; 27:1719-1742.

4. ESH-ESC Guidelines for the management of arterial hypertension. The Task Force for the management of arterial hypertension of the European Society of Hypertension
(ESH) and of the European Society of Cardiology (ESC). Eur Heart J. 2013; 34:2159-219.

5. ACE COMMITTI RECOMMENDATION. Recommendation for chamber quantification: a report from American Society of Echocardiography, s. J. Am. Soc. Echocardiogr. 2005; 18:1440-1463

6. Monyeki K., Kemperte I. The risc factors for elevated blood pressure and how to address cardiovascular risk factors: a review in pediatric populations. Journal of Human Hypertension. 2008; 22 (7):450-459.

7. Mancia G., Fagard R., Narkiewicz K. 2013 ESH-ESC Guidelines for the management of Hypertension. J. Hypertension. 2013; 31 (7): 1281-1357.
8. Patel M. S., et al. Serum parameters and echocardiographic predictors of death or need for transplant in newborns, children, and young adults with heart failure. Am. J. Cardiol. 2010; 105: 1798-1801.

9. Faris R.F. et al. Diuretics for heart failure. Cochrane. Database. Syst. Rev 2012; 2:CD003838.

10. Paulus W.J., van Dallegoij J.J. Treatment of heart failure with normal ejection fraction: an incovenient truth. J. Am. Coll. Cardiol. 2010; 55 (6):526-37.

11. Mary M., Stephens M.D., Lisa Maxwell M.D. Terapeutic options for the treatment of hypertension in children and adolescents. Clin. Respir. Pulm. Med. 2012; 6:13-25. 